\title{
Experimental measurements and theoretical model of the cryogenic performance of bialkali photocathode and characterization with Monte Carlo simulation
}

\author{
Huamu Xie, ${ }^{1}$ Ilan Ben-Zvi, ${ }^{2,3}$ Triveni Rao, ${ }^{2}$ Tianmu Xin, ${ }^{2}$ and Erdong Wang, ${ }^{2, *}$ \\ ${ }^{1}$ State Key Laboratory of Nuclear Physics and Technology, Institute of Heavy Ion Physics, \\ Peking University, Beijing 100871, China \\ ${ }^{2}$ Brookhaven National Laboratory, Upton, New York 11973, USA \\ ${ }^{3}$ Stony Brook University, Stony Brook, New York 11794, USA \\ (Received 16 November 2015; published 19 October 2016; corrected 27 October 2016)
}

\begin{abstract}
High-average-current, high-brightness electron sources have important applications, such as in highrepetition-rate free-electron lasers, or in the electron cooling of hadrons. Bialkali photocathodes are promising high-quantum-efficiency (QE) cathode materials, while superconducting rf (SRF) electron guns offer continuous-mode operation at high acceleration, as is needed for high-brightness electron sources. Thus, we must have a comprehensive understanding of the performance of bialkali photocathode at cryogenic temperatures when they are to be used in SRF guns. To remove the heat produced by the radio-frequency field in these guns, the cathode should be cooled to cryogenic temperatures. We recorded an $80 \%$ reduction of the $\mathrm{QE}$ upon cooling the $\mathrm{K}_{2} \mathrm{CsSb}$ cathode from room temperature down to the temperature of liquid nitrogen in Brookhaven National Laboratory (BNL)'s 704 MHz SRF gun. We conducted several experiments to identify the underlying mechanism in this reduction. The change in the spectral response of the bialkali photocathode, when cooled from room temperature $(300 \mathrm{~K})$ to $166 \mathrm{~K}$, suggests that a change in the ionization energy (defined as the energy gap from the top of the valence band to vacuum level) is the main reason for this reduction. We developed an analytical model of the process, based on Spicer's three-step model. The change in ionization energy, with falling temperature, gives a simplified description of the QE's temperature dependence. We also developed a 2D Monte Carlo code to simulate photoemission that accounts for the wavelength-dependent photon absorption in the first step, the scattering and diffusion in the second step, and the momentum conservation in the emission step. From this simulation, we established a correlation between ionization energy and reduction in the QE. The simulation yielded results comparable to those from the analytical model. The simulation offers us additional capabilities such as calculation of the intrinsic emittance, the temporal response, and the thickness dependence of the QE for the $\mathrm{K}_{2} \mathrm{CsSb}$ photocathode.
\end{abstract}

DOI: 10.1103/PhysRevAccelBeams.19.103401

\section{INTRODUCTION}

A high-average current beam is essential for linac-based light sources, electron-ion colliders, and electron cooling. To deliver a high average current requires both a high quantum-efficiency $(\mathrm{QE})$ photocathode, and high laser energy. Among the existing cathodes and drive lasers, to assure having a high QE, a low thermal emittance, and a long lifetime, many facilities choose the bialkali photocathode as their electron source [1-3].

In 1992, a collaboration of researchers from Los Alamos National Laboratory (LANL) and Boeing [4] demonstrated a record of $32 \mathrm{~mA}$ operation in a $433 \mathrm{MHz}$ normal

\footnotetext{
Corresponding author. wange@bnl.gov

Published by the American Physical Society under the terms of the Creative Commons Attribution 3.0 License. Further distribution of this work must maintain attribution to the author $(s)$ and the published article's title, journal citation, and DOI.
}

conducting photoinjector using a bialkali photocathode. In Cornell University's DC gun, a new record of $75 \mathrm{~mA}$ average current was achieved using a bialkali photocathode [5]; 1/e lifetime of the cathode was extrapolated to be about 2.6 days. BNL has two SRF guns with bialkali photocathodes. One is the $704 \mathrm{MHz}$ SRF gun, used as the prototype for the Energy Recovery Linac (ERL) [6], and the other is the $112 \mathrm{MHz}$ QWR SRF gun used for the proof-of-principle experiments for coherent electron cooling $(\mathrm{CeC})$ [7].

In contrast to the DC gun, in the SRF gun, both the normal conducting cathode and the cathode's support stalk are in a high rf field. The heat this field produces can reach up to hundreds of watts. The guns therefore, must be cooled by water or by liquid nitrogen. In BNL's $704 \mathrm{MHz}$ SRF gun, the calculated heat load on the cathode stalk is about 650 watts; it is dissipated by liquid nitrogen. In one experiment, we found that the $\mathrm{QE}$ of the $\mathrm{K}_{2} \mathrm{CsSb}$ photocathode dropped from $4 \%$ to $1 \%$ at $532 \mathrm{~nm}$ when it was cooled from room temperature to the temperature of liquid nitrogen, a significant loss of effectiveness. Hence, it is 
very important that we gain an understanding of the performance of the cathode at cryogenic temperatures and identify a means to mitigate this problem.

In looking at the cryogenic performance of other semiconductors, we concluded that several effects could cause this reduction in the electron yield: gas trapping at the cold surface [8], a reduction in electrical conductivity [9] due to the decreased density of carriers at cryogenic temperatures, or an increase in ionization energy (bandgap + electron affinity) due to a change in the structure of the lattice at low temperatures [10].

To better understand the contribution from each of these mechanisms, we undertook several experiments and developed an analytical model. We formulated a Monte Carlo PYTHON [11] code, based on a three-step model, to simulate the $\mathrm{QE}$ and its temperature dependence, temporal response, and the thermal emittance of the electron bunch. In the following sections, we describe our experimental arrangements and detail our results. We also derive an analytical expression to describe the temperature dependence of the spectral response. In addition, we develop the Monte Carlo code to simulate the spectral response and other properties. Finally, we compare our experimental results to our predictions.

\section{EXPERIMENTAL ARRANGEMENTS}

We systematically studied the $\mathrm{K}_{2} \mathrm{CsSb}$ photocathode in a dedicated photocathode material-deposition system.
This system consists of a transport suitcase, a storage chamber, a deposition chamber, a load-lock chamber, and three manipulators to transfer the sample between them. The substrate, on which the photocathode is deposited, is initially kept in the transport suitcase, and then transferred by a manipulator to the load-lock section. Utilizing two other manipulators, the substrate can be moved to the storage chamber, and the deposition chamber. Three sources, Cs, K, and $\mathrm{Sb}$, are mounted on the deposition chamber, each separated from one another and from the chamber by ultrahigh-vacuum (UHV) gate valves. The vacuum in the deposition chamber, pumped by an ion pump and a titanium sublimation pump, can attain the low level of $10^{-9} \mathrm{~Pa}$ and the vacuum in the three source arms can reach $10^{-8} \mathrm{~Pa}$. We employed a quartz crystal monitor to record the film's thickness during evaporation and utilized a residual gas analyzer (RGA) to analyze the partial pressure of the gases in the deposition chamber. Figure 1 provides a schematic diagram of this system. The cathode is irradiated by a $532 \mathrm{~nm}, 0.5 \mathrm{~mW}$ green laser, and the photocurrent leaving the cathode is monitored by a Keithley 6485 picoammeter [12].

The substrate is heated by a boron-nitride resistance heater, which is mounted beneath it, and it can be cooled by a cooling block with flowing cold nitrogen gas/liquid. The cooling rate is controlled by adjusting the flow rate of nitrogen. The lowest temperature which the substrate can reach is $166 \mathrm{~K}$, due to a limitation of the thermal contact between the cooling block and the cathode.

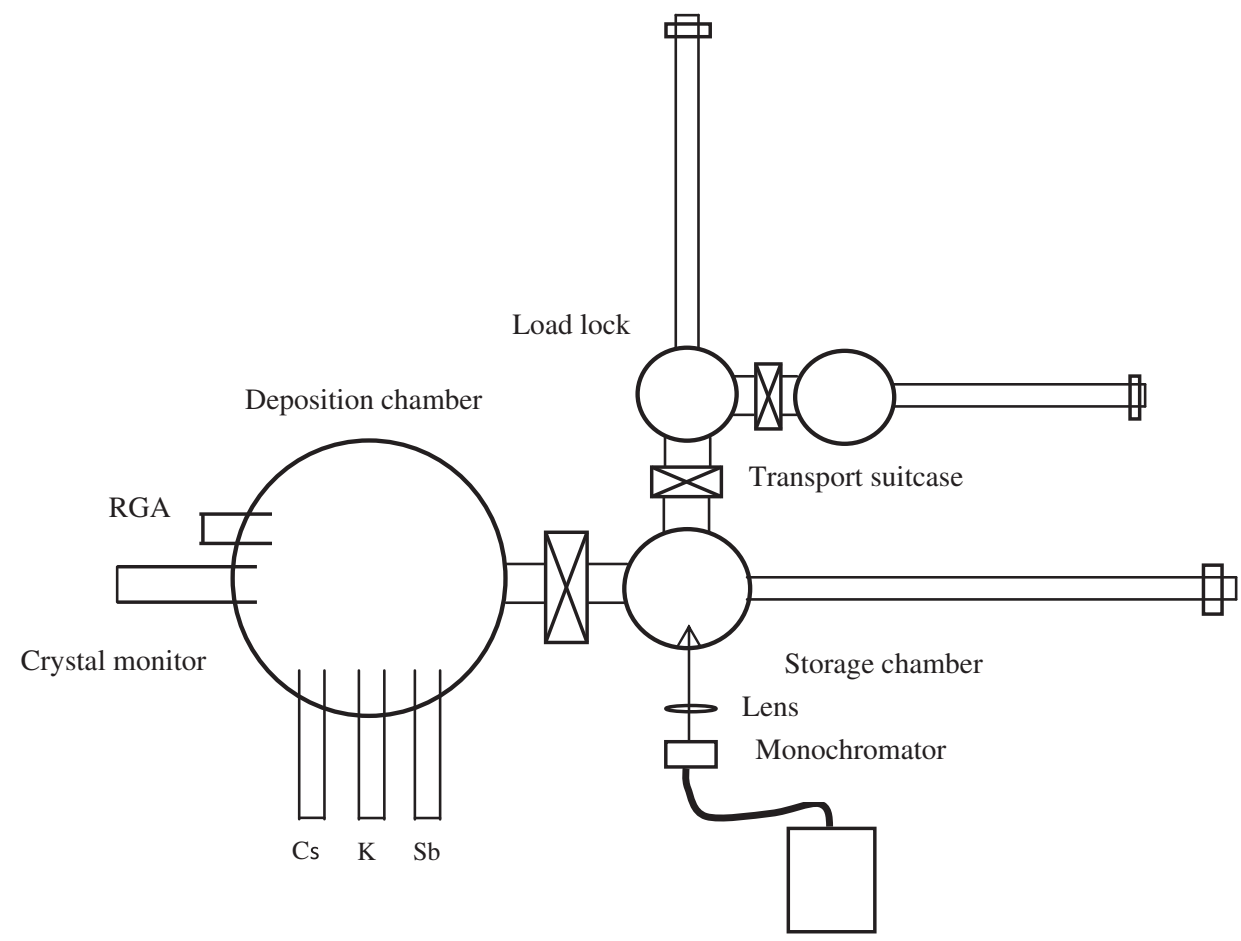

White light source

FIG. 1. A schematic of the BNL's $\mathrm{K}_{2} \mathrm{CsSb}$ photocathode deposition system. 
We grew a fresh $\mathrm{K}_{2} \mathrm{CsSb}$ photocathode on a molybdenum substrate following our standard procedure [12]. In the first step, the substrate was heated at $350{ }^{\circ} \mathrm{C}$ for approximately 6 hours to release the gas adsorbed on the surface; then it was held at $90^{\circ} \mathrm{C}$, ready for the cathode fabrication. During the cathode fabrication, we evaporated a $10 \mathrm{~nm}$ thick $\mathrm{Sb}$ layer on the substrate from an effusion cell at a rate of approximately $1 \AA / \mathrm{s}$; then, we raised the substrate's temperature to $130^{\circ} \mathrm{C}$, and deposited a $\sim 20 \mathrm{~nm}$ of potassium at an average rate of $0.6 \AA / \mathrm{s}$. At this time, the $\mathrm{QE}$ was measured as $\sim 5 \times 10^{-4}$. Then the heater was turned down in order for the substrate to be cooled at around $1{ }^{\circ} \mathrm{C} / \mathrm{min}$, while Cs was evaporated, and the QE increased steadily. When the photocurrent reached a plateau, the heater was turned off, and the substrate was cooled by flowing cold nitrogen. At this time the evaporation of $\mathrm{Cs}$ was reduced to keep the QE constant until the cathode cooled to $80^{\circ} \mathrm{C}$. Then the cathode was cooled quickly to room temperature by increasing the nitrogen flow. Using this procedure, we reproducibly fabricated cathodes with $\mathrm{QE}$ of $10 \%$ at $532 \mathrm{~nm}$.

The spectral response of the cathode was measured at the storage chamber using a monochromatic source derived from a white light. The light was transported via a fiber from the lamp to the cathode through a monochromator and vacuum viewport. The central wavelength of the light irradiating the cathode could be adjusted by the setting of the monochromator. The wavelength of the light was changed from 200 to $660 \mathrm{~nm}$ in $20 \mathrm{~nm}$ steps. We calibrated the optical power at these different wavelengths using a Newport 1930C power meter before and after measuring the cathode's spectral response. An electrometer was used to measure the photocurrent leaving the cathode with a biased voltage of $-50 \mathrm{~V}$. After we had measured the spectral response at room temperature, the cathode was slowly cooled from 300 to $273 \mathrm{~K}$. When the cathode's

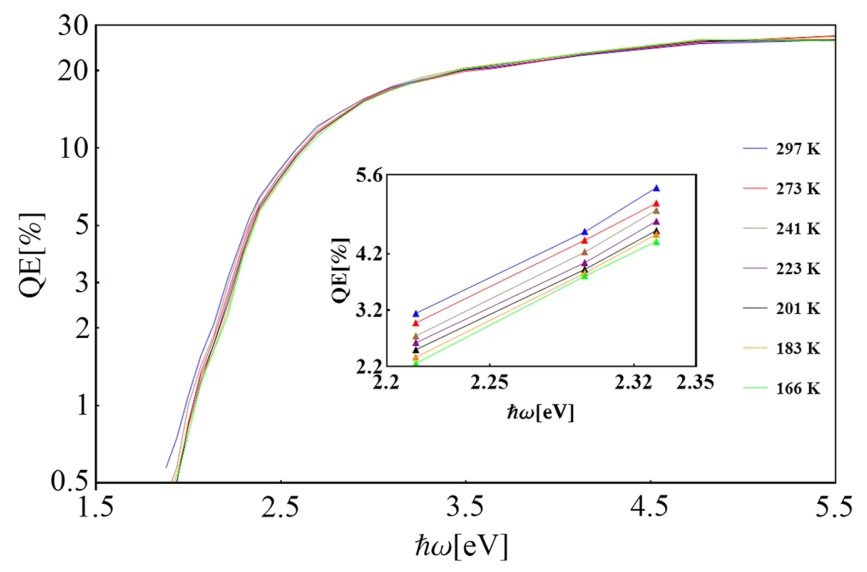

FIG. 2. The spectral response of $\mathrm{K}_{2} \mathrm{CsSb}$ cathode as it was cooled from room temperature to $166 \mathrm{~K}$. The inset is the zoomedin change in QE due to cooling; it dropped as the temperature was lowered.

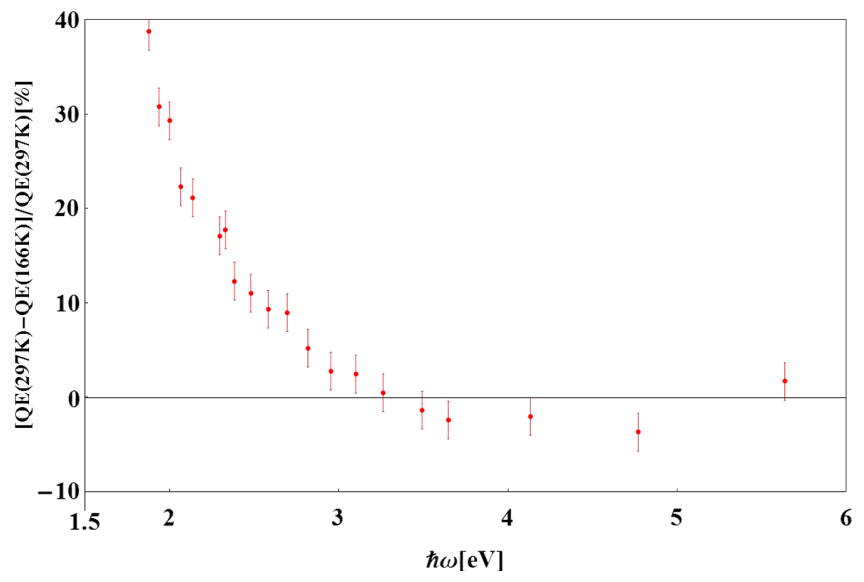

FIG. 3. The percentage of $\mathrm{QE}$ change from $\mathrm{RT}$ to $166 \mathrm{~K}$ as a function of the photon energy. The negative change is from the measurement errors.

temperature stabilized, we measured the spectral response by the same procedure. We then increased the flow of the liquid nitrogen, and repeated the procedure at 241, 223, 201,183 , and $166 \mathrm{~K}$. The measured photocurrents, together with the calibrated light power at different wavelengths, were used to calculate the $\mathrm{QE}$. The spectral response of the cathode at these seven different temperatures was obtained as shown in Fig. 2.

Figure 2 shows that about a $20 \%$ drop occurred at $532 \mathrm{~nm}$ from room temperature to $166 \mathrm{~K}$ from the insert, while Fig. 3 shows that QE drops more at lower photon energies. At higher photon energies, the QE experienced minimal change.

\section{RESULTS AND DISCUSSION}

The experiments detailed above showed that the QE decreased with the falling temperature. We discuss several plausible explanations for this reduction: gas trapping on the emission surface, change of the photocathode's electric conductivity, and the change in ionization energy.

\section{A. Gas trapping at the emission surface}

The cold surface of the cathode acts as a cryogenic pump and adsorbs the residual gas inside the chamber. The gas trapped on the cathode surface may increase the ionization energy and reduce the probability of the emission of electrons. To explore this effect, we illuminated the cathode at $166 \mathrm{~K}$ with a $0.5 \mathrm{~mW}$ green laser. The base pressure of the deposition chamber is $8 \times 10^{-11}$ Torr in room temperature. It reduces to $6.5 \times 10^{-11}$ Torr at LN2 cooling. Approximately $80 \%$ of the residual gas is hydrogen and $18 \%$ is nitrogen or $\mathrm{CO}$. The rest are $\mathrm{CO}_{2}$ and water. Both are 2 orders less than hydrogen. When illuminating the $0.5 \mathrm{~mW}$ laser, the baseline pressure went up to $5 \times 10^{-10}$ Torr. We observed the peak of $\mathrm{H}_{2}, \mathrm{~N}_{2}, \mathrm{H}_{2} \mathrm{O}$ increase. After 2 minutes, the pressure dropped down to 
$6.5 \times 10^{-11}$ Torr. However, the QE did not recover. The cathode was then brought to room temperature by the heater and then the QE was fully recovered, suggesting that if gas was adsorbed during cooling, it neither reacts with the cathode film nor damages it permanently. Hence, surface contamination by residual gas is not the reason for the reduction of the QE. With respect to the $704 \mathrm{MHz}$ SRF gun, in order to avoid the adsorption of gas on the cathode's surface, the gun was first cooled to the temperature of liquid helium, and the cathode was then inserted into the gun, while still being cooled by liquid nitrogen prior to making its final contact with the gun. The inner surface of the SRF cavity, held at a much lower temperature, then acts as a large cryogenic pump. Consequently, the surface of the liquid nitrogen cooled cathode would adsorb only a negligible amount of the residual gas.

\section{B. Change in electrical conductivity}

Other researchers have measured the temperature dependence of the conductivity of $\mathrm{K}_{2} \mathrm{CsSb}$ [9]. By fitting the curve from that data, we estimated that its conductivity is approximately $5.63 \times 10^{3} \mathrm{Ohm}^{-} \mathrm{m}$ at $166 \mathrm{~K}$. The maximum current that can flow through the crystal is derived using the following expression:

$$
I=A \kappa E_{0} / \varepsilon_{r}
$$

where $A$ is the emission area, $\kappa$ is the conductivity of $\mathrm{K}_{2} \mathrm{CsSb}, \varepsilon_{r}$ is the relative permittivity, and $E_{0}$ is the external field. The $\mathrm{K}_{2} \mathrm{CsSb}$ photocathode's relative permittivity, $\varepsilon_{r}$, is 7.87 [13], $E_{0}$ is about $500 \mathrm{~V} / \mathrm{m}$ in our equipment (the distance from the cathode to the anode is about $10 \mathrm{~cm}$, and the bias is $50 \mathrm{~V}$ ). Thus, we calculated that the upper limit of the current that can flow through the cathode is $0.35 \mathrm{~A}$, based on Eq. (1). The photocurrent we measured during the cooling process was $\sim 10 \mu \mathrm{A}$, which is far from the upper limit set by conductivity, indicating that conductivity is not the limiting factor for this current.

\section{Change in ionization energy}

The spectral response of the cathode at different temperatures shows that the $\mathrm{QE}$ depends strongly on temperature for photon energies below $3 \mathrm{eV}$. In the long wavelength regime, the QE declines with decreasing temperature. At $532 \mathrm{~nm}$, the QE of the cathode had dropped by $20 \%$ when it was cooled from room temperature $(300 \mathrm{~K})$ to $166 \mathrm{~K}$. The threshold of photon energy increases with decreasing temperature.

From Ref. [14] the spectral response of the QE can be expressed as follows:

$$
\mathrm{QE}(\omega) \approx \frac{1-R(\omega)}{1+\frac{\lambda_{\mathrm{opt}}(\omega)}{\lambda_{\mathrm{e}-\mathrm{e}}(\omega)}} \frac{\left(\hbar \omega-\emptyset_{\mathrm{eff}}\right)^{2}}{8 \emptyset_{\mathrm{eff}}\left(E_{F}+\emptyset_{\mathrm{eff}}\right)},
$$

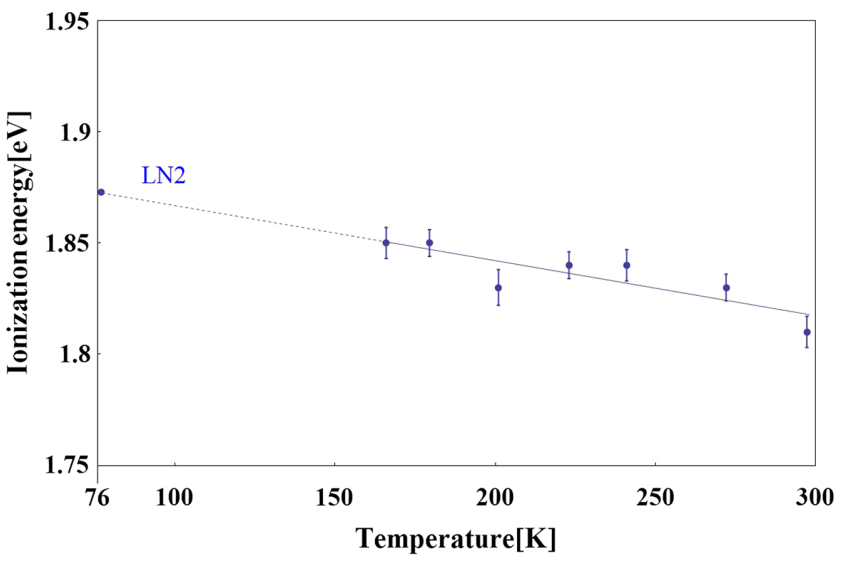

FIG. 4. Ionization energy, $E_{e}$, as a function of temperature.

where $R(\omega)$ is the cathode optical reflectivity, $\varnothing_{\text {eff }}$ is the effective work function of the cathode, $E_{F}$ is the Fermi energy of the cathode, $\lambda_{\text {opt }}(\omega)$ is the photon absorption length, and $\lambda_{\mathrm{e}-\mathrm{e}}(\omega)$ is the electron-electron mean-free path.

The ionization energy for the $\mathrm{K}_{2} \mathrm{CsSb}$ cathode is given by the intercept with the photon energy axis in the plot of the square root of the QE versus the photon energy $\hbar \omega$. (Here, we used the ionization energy to substitute for the effective work function.) For the data shown above, we determined that the ionization energy increases by $0.03 \mathrm{eV}$ when the cathode is cooled to $166 \mathrm{~K}$.

The measured ionization energy as a function of temperature is plotted in Fig. 4. In order to get the ionization energy of $77 \mathrm{~K}$, we fitted the results with a linear equation. The best fit of the linear equation can be expressed as

$$
E_{e}=1.89-0.00025 T
$$

where $T$ is the temperature of the cathode (in $K$ ). Using Eq. (3), the ionization energy at $300 \mathrm{~K}$ and at $77 \mathrm{~K}$ can be calculated as 1.81 and $1.875 \mathrm{eV}$, respectively.

Spicer [15] found that the $\mathrm{QE}$ of the $\mathrm{Na}_{2} \mathrm{CsSb}$ cathode at visible and IR wavelengths degraded when the cathode was cooled to the temperature of liquid nitrogen. Cultrera [16] also observed such behavior when the $\mathrm{Cs}_{3} \mathrm{Sb}$ photocathode was cooled to $90 \mathrm{~K}$. Electrons in the donor levels near the Fermi level of the semiconductor can be emitted when they absorb photons with an energy lower than the sum of band gap and the electron affinity. According to Spicer, at cryogenic temperatures, there are fewer donor-level electrons. Hence, the QE of the bialkali photocathode would drop as the cathode is cooled [15].

The difference between Spicer's finding and our experiment is that we observed a reduction in the $\mathrm{QE}$, even for photon energies larger than the ionization energy. Therefore, some other mechanisms besides the reduction in number of electrons in donor states should be considered.

To explain the experimental results, we developed two models to characterize the temperature dependence of the 
QE for the $\mathrm{K}_{2} \mathrm{CsSb}$ photocathode, based on Spicer's threestep model [15]. An analytical model was developed to explain the general temperature dependence of the QE, and a simulation program was written to give a more precise description of the photoemissive mechanism of the bialkali photocathode and at the same time to describe the cooling effect on the bialkali photocathode.

\section{ANALYTICAL MODEL}

We started with the fundamental assumption that, for a given cathode, only the ionization energy changes as a function of temperature. This change could be caused either by the change in the band gap due to an alteration in the lattice, or a change in electron affinity at the surface. The following additional assumptions were made to simplify the model. 1. The photon reflection of the surface was not considered, and all the transmitted photons are assumed to be absorbed. So the initial photon number of the model is defined by the photon penetrate into the crystal. 2. Multiphoton excitation is not considered due to its very low probability. 3. The probability distribution of the electron energy in the valence band is assumed to be Maxwell-Boltzmann [17], with a cutoff at the top of the valence band. This is a first order simplification. A more accurate distribution is used in the Monte Carlo simulation. 4. The probability distribution of the electron energy in the conduction band after excitation is similar to that in the valence band, but with a tail caused by collisions in the conduction band. Because our experiment laser power is in the $\mu \mathrm{W}$ level, the electron density in the excited state is very low. Thus the conduction band has sufficient states to accept the excited electrons. Due to electron phonon scattering, a fraction of electrons will lose or gain the energy and diffuse to both the high energy side and the low energy side giving rise to a tail as described by the Maxwell-Boltzmann distribution. 5. According to [18], the density of states in the topmost valence band has a half width half maximum of $\sigma / 2$. 6. Scattering or collision is not considered explicitly during the transport step. However, the tail in the density distribution and the constant $C$ in Eq. (11) takes these effects into consideration implicitly. 7. As a first order approximation, the energy is conserved. Momentum conservation is not considered here. Both energy and momentum conservation are applied in Monte Carlo simulation.

The first step in the three-step model is photon absorption. The laser power inside the cathode decays exponentially, due to absorption of photons by the cathode material. The laser's power $I(z)$ at a depth $\mathrm{z}$ from the surface can be expressed as

$$
I(z)=I_{0} e^{-\alpha(\hbar \omega) z},
$$

where $I_{0}$ is the incident light power, $\hbar \omega$ is the photon energy, and $\alpha(\hbar \omega)$ is the absorption coefficient of the
$\mathrm{K}_{2} \mathrm{CsSb}$ at the photon energy $\hbar \omega$. So, the fraction $P_{\mathrm{abs}}$ of laser power absorbed by the cathode with a thickness of $L(\mathrm{~nm})$ can be written as the integral of $I(z)$ :

$$
P_{\mathrm{abs}}=\frac{\int_{0}^{L} I(z) d z}{I_{0}}=1-e^{-\alpha(\hbar \omega) L} .
$$

Based on the absorption data for the $\mathrm{K}_{2} \mathrm{CsSb}$ material at different wavelengths in Ref. [19], we derived an analytical expression for $\alpha(\hbar \omega)$ (valid for $\hbar \omega<4 \mathrm{eV}$ ):

$$
\alpha(\hbar \omega)=19.82-27.95 \hbar \omega+11.15(\hbar \omega)^{2} .
$$

Thus, if the number of the incident photons (photon energy is $\hbar \omega$ ) is $N_{p}$, the total number of the photons absorbed by the electrons is

$$
N_{p}\left(1-e^{-\alpha(\hbar \omega) L}\right) .
$$

The electrons in the valence band absorb the photons, and those with energy higher than the band gap are excited to the conduction band. Figure 5 shows the band structure and the electron-energy probability distribution inside the $\mathrm{K}_{2} \mathrm{CsSb}$. From the electron energy density of states given in Ref. [18], the spread of electron energy in the valence band $\sigma$ is $\sim 1.2 \mathrm{eV}$. When the photon energy is higher than the sum of the band gap and $\sigma$, all the electrons will be excited to the conduction band. The probability distribution and the energy spread of these excited electrons will be the same as those in the valence band. If the photon energy is lower than the sum of band gap plus $\sigma$ (Fig. 5), the distribution is the Maxwell-Boltzmann distribution with a cutoff.

We consider only the process wherein the electrons absorb the photons, and are excited from the valence band

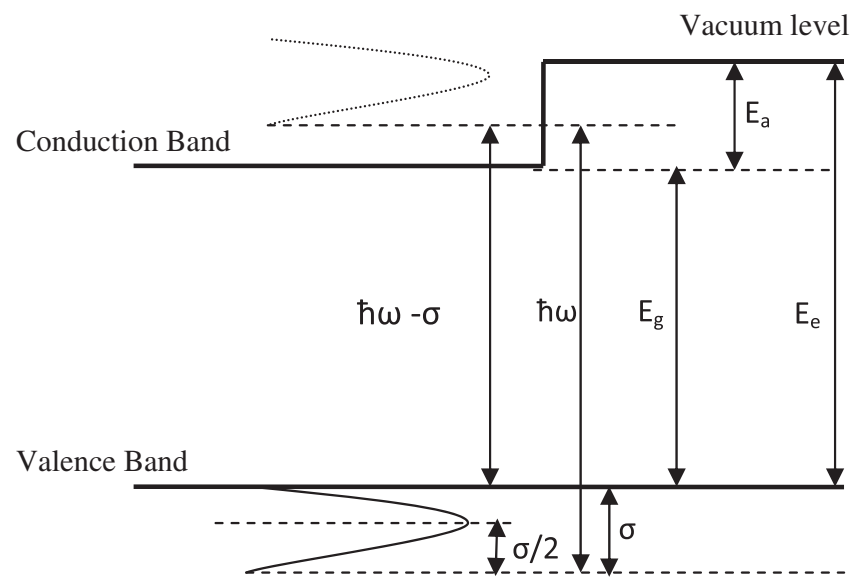

FIG. 5. The probability distribution of the electrons in the band structure of the crystal. The solid parabola is the electron energy probability distribution in the valence band, while the dashed parabola curve is the electron energy probability distribution after being excited to the conduction band. $\sigma$ is the width of the band below the maximum of the valence band. 
into the conduction band; then, the distribution on the conduction band can be simplified by a MaxwellBoltzmann energy distribution [17],

$$
f(E) \mathrm{d} E=2 \sqrt{\frac{E}{\pi}}\left(\frac{1}{k_{B} T}\right)^{3 / 2} \mathrm{e}^{-\frac{E}{k_{B} T}} \mathrm{~d} E,
$$

where $E$ is the energy of the electron above the minimum of the conduction band (the energy zero point starts from the bottom of the conduction band), and $k_{B} T / 2$ is the most probable energy. The spread of electron energy in the valence band is $\sigma$ with the maximum likelihood at $\sigma / 2$. After electrons are excited above the conduction band, the value of $\sigma$ for the distribution has not changed. Thus, we replaced $k_{B} T$ by $\sigma$ in the following calculation.

Meanwhile, we moved the relative energy level from the bottom of the conduction band to the top of the valence band. Thus, the modified Maxwell-Boltzmann energy distribution can be written as

$$
\begin{aligned}
& f[E-(\hbar \omega-\sigma), \sigma] \mathrm{d} E \\
& \quad=2 \sqrt{\frac{E-(\hbar \omega-\sigma)}{\pi}}\left(\frac{1}{\sigma}\right)^{3 / 2} \mathrm{e}^{-\frac{E-(\hbar \omega-\sigma)}{\sigma}} \mathrm{d} E,
\end{aligned}
$$

where $E$ is the energy of the electron. Since our model does not consider explicitly electron scattering during transport, and it is assumed to be independent of temperature, the probability distribution of the electron energy in the cathode is not changed when the electrons reach the emission surface.

Here the ionization energy $E_{e}(T)$ can be expressed as

$$
E_{e}(T)=E_{e}(0)+\varphi(T),
$$

where $E_{e}(0)$ is the ionization energy at room temperature, which equals the sum of band gap energy $E_{g}$ and the electron affinity $E_{a}$ at room temperature. $\varphi(T)$ was set as zero at room temperature. The temperature has an effect on the crystal's lattice and its surface properties, and hence, changes the energy band gap or electron affinity [10]. $\varphi(T)$ can be due to the temperature dependence of either the band gap, or in the electron affinity.

Only those electrons with energy higher than the ionization energy $E_{e}$ can be emitted. The emission probability $P_{\text {emit }}$ of the electron can be written as

$$
P_{\mathrm{emit}}=C\left(\int_{E_{e}}^{+\infty} f[E-(\hbar \omega-\sigma), \sigma] d E\right) .
$$

$C$ is a constant accounting for electrons that are either trapped in the cathode material, or diffuse back to the substrate, which is related to the second step, and is considered not to change with temperature in this model. The number of electrons that can be emitted is expressed by

$$
\begin{aligned}
N_{e} & =N_{p}\left(1-e^{-\alpha(\hbar \omega) L}\right) P_{\mathrm{emit}} \\
& =N_{p} C\left(1-e^{-\alpha(\hbar \omega) L}\right)\left(\int_{E_{e}}^{+\infty} f[E-(\hbar \omega-\sigma), \sigma] d E\right),
\end{aligned}
$$

where $N_{p}$ is the number of photons. Since the QE of the photocathode at photon energy $\hbar \omega$ can be expressed as the ratio of the number of emitted electrons over the number of incident photons

$$
Q E=\frac{N_{e}}{N_{p}},
$$

then Eq. (13) can be written as

$\mathrm{QE}=C\left(1-e^{-\alpha(\hbar \omega) L}\right)\left(\int_{E_{e}}^{+\infty} f[E-(\hbar \omega-\sigma), \sigma] d E\right)$

and Eq. (14) can be transformed to

$\mathrm{QE}=C\left(1-e^{-\alpha(\hbar \omega) L}\right)\left(1-\int_{0}^{E_{e}(0)+\varphi(T)-(\hbar \omega-\sigma)} f(E, \sigma) d E\right)$.

Since $\varphi(T)$ critically depends on the cathode material and the growth process, it should be a fitting parameter for a given cathode. With the equations above, and using the constants and parameters listed in Table I, we determined the temperature dependence of the ionization energy, $E_{e}$, using the following steps. 1. Calculate $\mathrm{QE}$ at room temperature at $532 \mathrm{~nm}$, and compare it to the measurement to

TABLE I. Parameters used to calculate QE from Eq. (15).

Value

Absorption coefficient, $\alpha(\hbar \omega)(\hbar \omega<4 \mathrm{eV})$

Cathode's thickness, $L$

$\alpha(\hbar \omega)=\left[19.82-27.95 \hbar \omega 11.15(\hbar \omega)^{2}\right] \mathrm{cm}^{-1}[19]$
$40 \mathrm{~nm}$
$2-5.5 \mathrm{eV}$

Photon energy, $\hbar \omega$

$2-5.5 \mathrm{eV}$ 


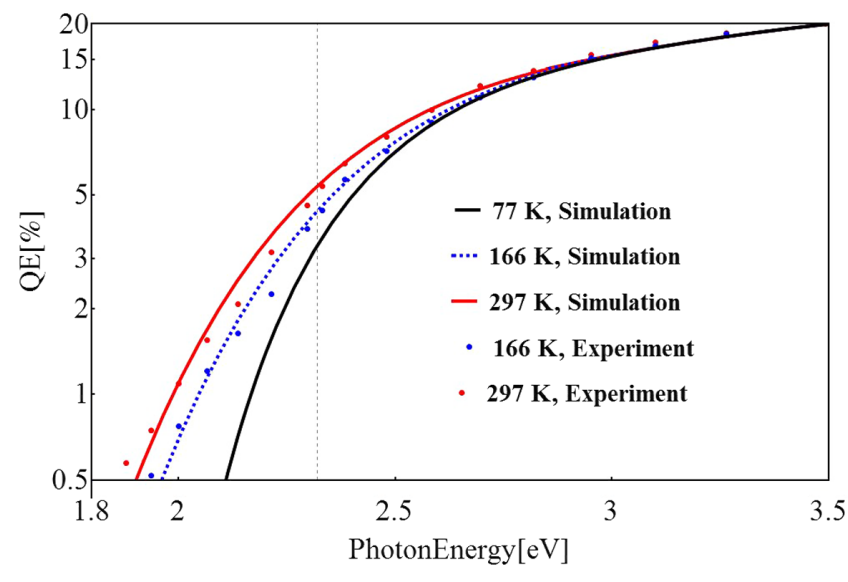

FIG. 6. The calculated curve fits with the measured spectralresponse data at room temperature (red line), and at $166 \mathrm{~K}$ (blue, dashed). The $R^{2}$ of these two nonlinear fits are 0.99 . The black curve is the expected spectral response of the cathode at $77 \mathrm{~K}$. The vertical dashed line shows the QE change at $532 \mathrm{~nm}$.

determine $C$. 2. Calculate the spectral dependence at room temperature, and use $E_{e}$ as the fitting parameter to match theoretical prediction to the experimental measurement. 3. Given these values of $C$ and $E_{e}(0)$, calculate the spectral dependence for each temperature with a single fitting parameter, $\varphi(T)$.

From the fitting to the experimental results, the best fit for $\varphi(T)$ can be expressed as follows:

$$
\varphi(T)=-0.0003 T \text {. }
$$

The fitted $\varphi(T)$ at $166 \mathrm{~K}$ is about $0.05 \mathrm{eV}$, very close to the value of $0.04 \mathrm{eV}$ calculated from Eq. (3).

Both the model and the experimental data suggest that for photon energies higher than $3 \mathrm{eV}$, the $\mathrm{QE}$ is independent of temperature. Although we completed the data analysis for all the measured temperatures, for clarity we show just two of the experimental data sets, along with the analytical results in Fig. 6. This fit confirms that the change in $E_{e}$ with temperature is the dominant mechanism for reducing the electron yield at lower temperatures, especially for photon energies close to $E_{e}$.

$E_{e}$ was expected to increase by $0.15 \mathrm{eV}$ when the cathode has been cooled to the temperature of liquid nitrogen based on Ref. [20]. By using this analytical model, when irradiated with a $532 \mathrm{~nm}$ laser, a 50\% drop in $\mathrm{QE}$ could be expected when the cathode is cooled from room temperature to that of liquid nitrogen. However, when we measure the $\mathrm{QE}$ of another $\mathrm{K}_{2} \mathrm{CsSb}$ cathode in the $704 \mathrm{MHz}$ SRF gun at the temperature of liquid nitrogen, we observed an $80 \%$ drop in the QE. We attribute this discrepancy to the simplifications we made to the threestep model, especially our exclusion of the scattering process in the transport step, and the conservation of momentum.

\section{MONTE CARLO SIMULATION}

The analytical model explained the trend of $\mathrm{QE}$ while the cathode is being cooled down. However, it does not incorporate the change in electron energy due to scattering during the transport of electrons to the cathode surface. Thus, to understand the effect of these various scattering events, we developed a Monte Carlo code for simulating more precisely the photoemission with the threestep model.

The first step in the simulation is photon absorption. When the cathode is illuminated by the laser, the absorbed energy decays exponentially along the cathode's thickness, as described in Eq. (4). The absorption coefficient we used here is interpolated from Ref. [13]. The electrons occupying the valence band absorb the photon energy and can only be excited when there is an empty state above the minimum of the conduction band with the same energy as the photon energy plus the initial energy of the electron. To calculate this, we generated the discrete electron density of states at energy $E_{i}$, with a resolution of $0.01 \mathrm{eV}$, matching the continuous density of states (DoS) of the $\mathrm{K}_{2} \mathrm{CsSb}$ semiconductor from Ref. [13] where the density of states (DoS) for $\mathrm{K}_{2} \mathrm{CsSb}$ has been calculated with the localized spherical-wave method. The initial electron exciting conditions are based on the DoS of $\mathrm{K}_{2} \mathrm{CsSb}$ below the maximum of valance band. A function $N(E)$ was used to interpolate the DoS of $\mathrm{K}_{2} \mathrm{CsSb}$ from -2 to $5 \mathrm{eV}$, based on the data in Ref. [18]. The number of the electrons, $n_{i}$, with energy $E_{i}$, in the conduction band can be determined by the convolution of the DoS in the valance band and in the conduction band. Equation (17) shows the number of excited electrons with discrete energy $E_{i}$ :

$$
n_{i}=N_{0} \frac{N\left(E_{i}\right) N\left(E_{i}-\hbar \omega\right) \Delta E}{\int_{E_{g}}^{\hbar \omega} N\left(E_{i}-\hbar \omega\right) N\left(E_{i}\right) d E_{i}},
$$

where $N_{0}$ is the initial number of photons, $\hbar \omega$ is the photon's energy, $E_{i}$ is the energy of the electron in the conduction band after absorbing the photon energy, and $\left(E_{i}-\hbar \omega\right)$ is the energy of the electron in the valence band. The denominator of the Eq. (17) is the normalization factor. Figure 7 shows the distribution of the generated electrons.

In the second step, the electrons will diffuse with the initial velocity and then experience three possible types of scattering: Electron-electron scattering, electron-hole scattering, and electron-phonon scattering. Here we did not consider the electron-hole recombination in electron transport.

In the photoemission process, electron-electron scattering could occur when the energy of the electron is higher than $2 E_{g}$. The electrons in the conduction band can scatter off an electron in the valence band, and the electrons in the 

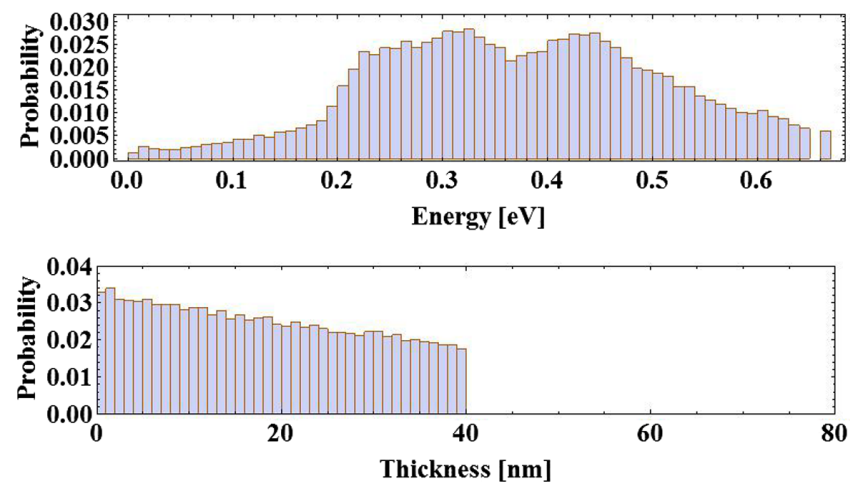

FIG. 7. (top) The histogram of the electron-energy distribution in the conduction band excited by $2.32 \mathrm{eV}$ laser photons at room temperature; the electron energy is $\left(\hbar \omega-E_{g}\right)$ with a resolution of $0.01 \mathrm{eV}$. The $Y$ axis is the probability distribution of electrons at different energies. (bottom) The longitudinal distribution of excited electrons along the cathode's thickness $(40 \mathrm{~nm})$ with exponential decay.

valence band then absorb sufficient energy to be excited above the conduction band. Figure 8(a) shows the e-e scattering process. The e-e scattering's mean-free path of the bialkali material was obtained from Spicer's paper [15]. If the energy of electrons is higher than the minimum of the conduction band by n $E_{g}$, it is possible that e-e scattering can happen up to $\mathrm{n}$ times, where $\mathrm{n}$ is an integer. The probability of scattering is then given by the single scattering probability raised to the power $n$. During the electron-electron scattering process, the electron in the conduction band would lose more energy than the band gap so as to excite the electron in the valence band to the conduction band. Since the width of the outermost valence

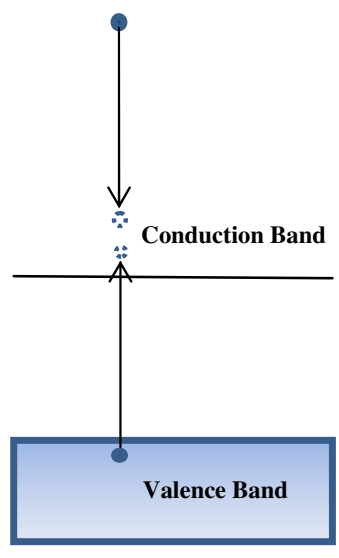

Electron-electron scattering

(a)
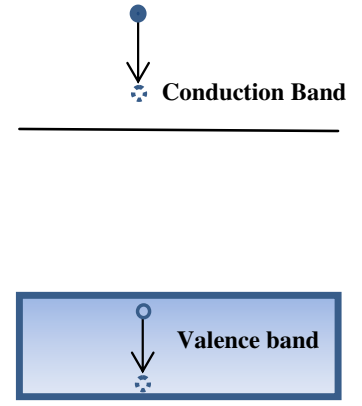

Electron-hole scattering

(b)
FIG. 8. Electron-electron scattering (a), and electron-hole scattering (b). The solid/ring circles are the initial states of electrons/holes respectively, and the dotted rings are their end states after scattering. band is $1.2 \mathrm{eV}$, the scattering process is not allowed wherein the electron in the conduction band loses energy to occupy the valence band, and simultaneously excites a valence band electron to a higher level within the valence band. The range of change in electron-electron scattering energy can extend from $E_{g}$ to $E_{g}+1.2 \mathrm{eV}$.

In a semiconductor photocathode, $p$-doping can lower the Fermi level as well as that of the vacuum level, which helps to increase the QE. The overcesiated bialkali photocathode could result in $p$-doping, and then the Fermi level could lie closer to the maximum of the valence band, enabling holes to exist in the valence band [21]. The highenergy electrons in the conduction band scatter with a low energy electron in the valence band, and bring up an electron from the bottom of the valence band to fill the hole on top, and leave a hole at the bottom. Using another point of view, this can be seen as the scattering of a high-energy electron off the hole. So during the scattering process, the electron will lose energy in the range of zero to $1.2 \mathrm{eV}$ (the width of the valence band from the DoS of $\mathrm{K}_{2} \mathrm{CsSb}$ [18]) to the hole. Figure 8(b) is a schematic of electron-hole scattering.

Electron-phonon scattering happens when electrons are scattered from one energy minimum to another during their interaction with lattice vibrations. During this process, the electrons may absorb or emit an optical phonon. The phonon energy was estimated to be about $0.027 \mathrm{eV}$ [17] at room temperature. Electron-phonon scattering will stop once the energy of the electron is less than one phonon energy above the minimum of the conduction band.

A list of parameters has to be generated for the transport step, including the longitudinal and transverse position, longitudinal and transverse velocity, and energy. The energy of the electron was generated, as described in Eq. (17). The magnitude of the velocity of the electrons can be calculated from the energy of the electron using its average effective mass that was taken as $0.1175 m_{0}$ based on Ref. [22],

$$
m^{*}=\frac{E_{g}}{R_{\infty}} m_{0}
$$

where $R_{\infty}$ is the Rydberg constant, $13.606 \mathrm{eV}$, and $m_{0}$ is the free electron's mass. The effective mass is an average over values the electron can take on within the calculated band structure. The direction of the velocity is randomly generated uniform distribution. The transverse position of the electron is also a randomly generated uniform distribution inside the size of the laser spot (the diameter of the laser spot used here was $2 \mathrm{~mm}$ ). The longitudinal position of the electrons is randomly generated along the cathode's thickness with an exponential distribution as the laser power decays. We neglected the influence of the laser's arrival time on the longitudinal distribution. Figure 7, bottom, shows the particles' distribution along the cathode's 
TABLE II. Parameters used in the Monte Carlo simulation.

\begin{tabular}{lc}
\hline \hline Parameters & Value \\
\hline Electron-phonon scattering MFP & $3 \mathrm{~nm}$ \\
Thickness & 1 to $300 \mathrm{~nm}$ \\
Photon number & 100,000 \\
Photon energy range & 1.6 to $5.5 \mathrm{eV}$ \\
Phonon energy in $\mathrm{K}_{2} \mathrm{CsSb}$ & $0.027 \mathrm{eV}$ \\
Band gap & $1.2 \mathrm{eV}$ \\
\hline \hline
\end{tabular}

thickness. Based on the above process, we obtained the initial parameters of the electrons in the conduction band that we needed for the transport calculation in the second step.

The scattering probability we used here is calculated as follows:

scattering probability $=\frac{\text { time step } \times \text { electron velocity }}{\text { MFP }}$.

The time step was set a factor of 5 less than the minimum of the three scattering mean-free paths (MFPs), divided by the mean velocity of the electrons. The mean-free path of electron-electron scattering and electron-hole scattering, which are energy dependent, are derived from Ref. [15]. Table II shows the mean-free path of electron-phonon scattering. Thus, all three scattering processes are represented accurately.

The electrons' energy will change according to the laws of energy conservation during the scattering process. The direction of the movement of electrons after scattering will change randomly. A random angle was generated after each scattering. Hence, the velocity vector of the electrons after each scattering can be obtained based on the energy change in the collisions, and the random velocity distribution.

By repeating the scattering process above, a fraction of the electrons excited by the photons will arrive at the cathode's emission surface. If the energy of the electron at this point is lower than the electron affinity, it would be reflected back and continue the diffusion process in the conduction band. If the electron reaches the surface with energy higher than the electron affinity, it can be emitted into the vacuum. In this step, both the energy and momentum conservations are taken into consideration, as shown in Fig. 9. Equations (20) and (21) show the momentum-conservation condition:

$$
\begin{gathered}
P_{\text {Tran }}^{\text {In }}=P_{\text {Tran }}^{\text {Out }} \\
P^{\text {Out }}=\sqrt{2 m\left(E-E_{a}+E_{\text {sch }}\right)},
\end{gathered}
$$

where $E$ is the energy of the electron before being emitted, $E_{a}$ is the electron affinity, and $E_{\text {sch }}$ is the reduction due to

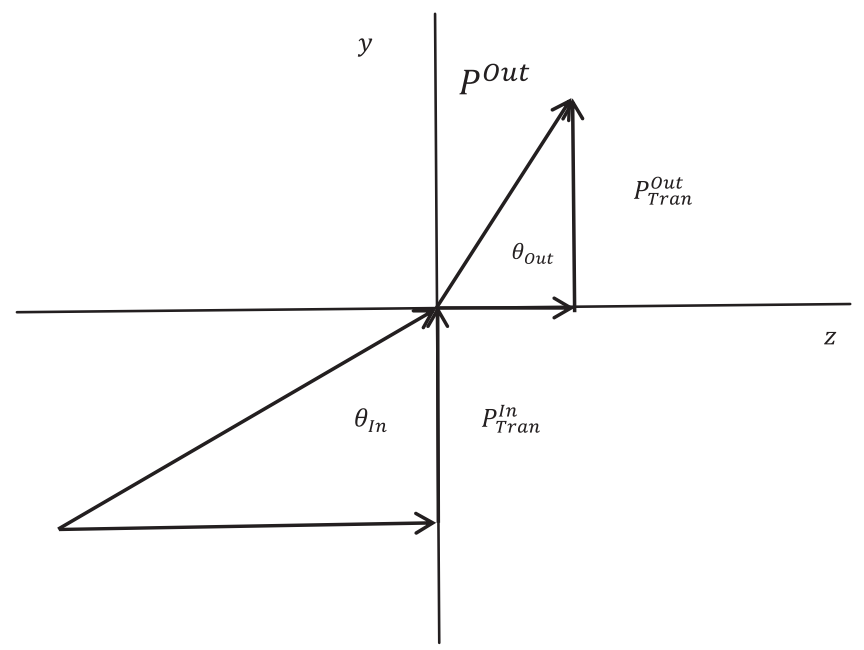

FIG. 9. The transverse momentum conservation diagram; the $y$ axis is the cathode surface-vacuum interface, and the $\mathrm{z}$ axis is the direction of electron emission. There is a maximum value for $\theta_{\text {In }}$ for the electrons to be emitted out.

the Schottky effect. In our simulation, the Schottky effect is not considered.

The transverse momentum conservation condition in the simulation is shown as Eq. (22),

$$
P^{\text {Out }}>P_{\text {Tran }}^{\text {out }}
$$

Those electrons with energy higher than the electron affinity and transverse momentum conserved will be emitted into the vacuum. Once there, the effective mass of the electron is replaced by the rest mass for calculating the emittance. Electrons that were not emitted will be reflected back, and continue to scatter with other electrons or phonons. They still will have some probability to be emitted, if the emitting condition is satisfied. A metal substrate $(\mathrm{Mo}, \mathrm{Cu}$, or $\mathrm{Cr}$ ) is used as the cathode's substrate. The Fermi level of the metal is aligned to the Fermi level of the semiconductor. Thus, electrons that diffuse back to the substrate could be absorbed totally by the substrate without any reflection. When the energy of all electrons inside the cathode is less than one phonon energy, the simulation is terminated.

The QE is expressed as

$\mathrm{QE}=\frac{\text { number of electrons emitted out of cathode }}{\text { number of incident photons }}$.

The intrinsic thermal emittance, normalized to the spot size $\sigma_{y}$ of the laser, was calculated using Eq. (24),

$$
\frac{\varepsilon_{n}}{\sigma_{y}}=\frac{\sqrt{\left\langle p_{y}{ }^{2}\right\rangle}}{m c},
$$




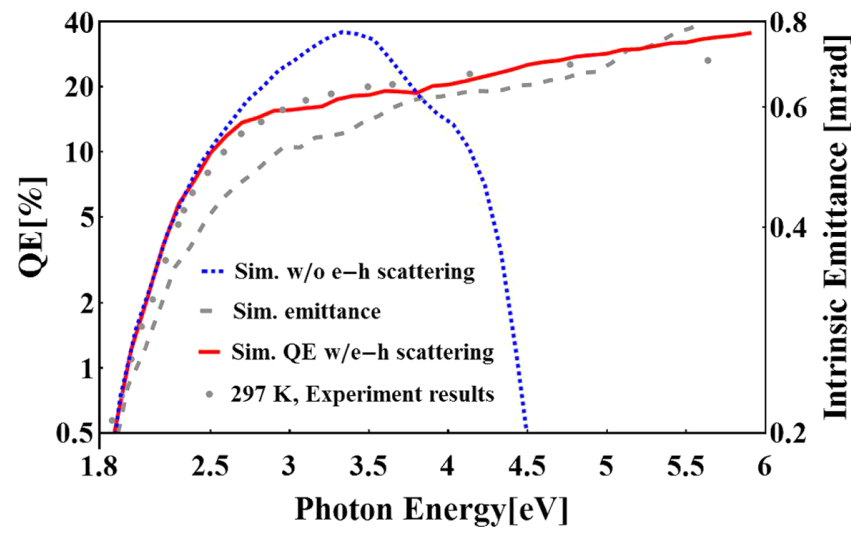

FIG. 10. The results of our simulation of spectral response with and without electron-hole (e-h) scattering, and thermal emittance at room temperature. The dots are the experimental results of the spectral response of the $\mathrm{K}_{2} \mathrm{CsSb}$ photocathode at room temperature.

where $\frac{\varepsilon_{n}}{\sigma_{v}}$ is the cathode thermal emittance (per unit of spot size) with the unit of $\mathrm{mm}-\mathrm{mrad} / \mathrm{mm}$. We ran the code with the parameters listed in Table II, and scanned the electron affinity to fit the spectral response of $\mathrm{K}_{2} \mathrm{CsSb}$ at room temperature. The postprocessing function was able to scan the photon energy and thus obtained the spectral response shown in Fig. 10.

The simulation for different photon energies, together with a fitted value of the ionization energy of $1.7 \mathrm{eV}$, matches very well our experimental data at room temperature. The smaller ionization energy is caused by the distribution of electron energy used in the simulation. In the simulation, the initial distribution of the electrons is derived from the density of states; in the analytical model we used the Maxwell-Boltzmann distribution as the starting condition.

It is important to note the difference in the simulation results when electron-hole scattering is also included. In contrast to the expected reduction in $\mathrm{QE}$ for $\hbar \omega>2 E_{g}$, both the simulation and our experimental results indicate a continued increase in the QE. For the bialkali photocathode fabricated in our experiment, the last step is evaporating the Cs until the QE reaches a plateau. This process could lower the vacuum level in this semiconductor, and result in the heavy $p$-doping of it. Once the Fermi level lies below the valence band, there are holes below the maximum of the valence band, and the scattering of electron and holes should be considered. If electron-electron scattering alone is taken into account in the QE calculation, for $\hbar \omega>2 E_{g}$, the QE would drop dramatically. If both electron-electron and electron-hole scatterings are considered, the spectral response in this range would be significantly different. Since in electron-electron scattering, the minimum energy loss from an excited electron is the band gap energy, while the maximum energy loss in an electron-hole scattering is $1.2 \mathrm{eV}$, the rate of energy loss in the former process is much

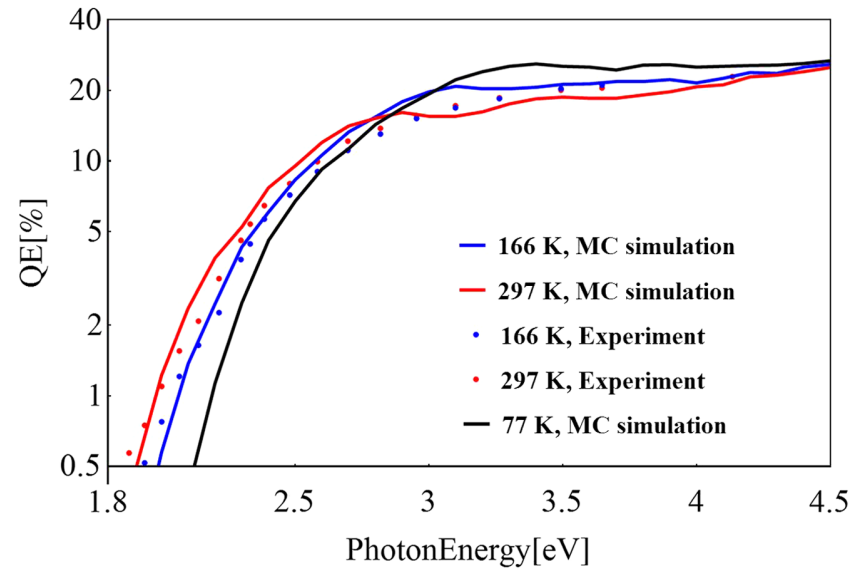

FIG. 11. Simulated spectral response of the bialkali photocathode at room temperature $(297 \mathrm{~K}), 166 \mathrm{~K}$, and liquid-nitrogen temperature (solid lines) and experimental data at room temperature $(297 \mathrm{~K})$ and $166 \mathrm{~K}$ (dots).

larger than that in the latter. Hence, if electron-hole scattering is taken into consideration in the simulation, the QE continues to increase, even for $\hbar \omega>2 E_{g}$.

The simulated result of thermal emittance at $532 \mathrm{~nm}$ is $0.4 \mathrm{~mm}$-mrad for a $1-\mathrm{mm}$ spot size, which is smaller than Cornell's measurement (about $0.56 \mathrm{~mm}-\mathrm{mrad} / \mathrm{mm}$ ) [23]. This difference could be attributed to the effect of the surface roughness of the cathode on the emittance in the experiment, which was not considered in the simulation. It also could be attributed to the different laser transverse distribution. We are using uniform transverse distribution in our simulation and Cornell used Gaussian distribution with a cutoff radius in their laser system [24]. With increasing mean transverse energy, the thermal emittance would increase when the energy of the illuminated photons becomes larger.

Based on prior literature [20], the band gap of $\mathrm{K}_{2} \mathrm{CsSb}$ increases with the lowering of temperature. To determine the $\mathrm{QE}$ as a function of temperature, we used the band gap as a fitting parameter. We found no published data on the temperature dependence of phonon energy and the probability of electron-phonon scattering; hence, we still used the phonon energy corresponding to room temperature. Figure 11 shows the spectral response curves obtained by simulations and experiments. The change in band gap energy we used in the simulation is derived from Ref. [25]. As depicted, the QE at the long-wavelength end of the spectrum decreases with decreasing temperature and increasing band gap, matching our experimental observations. At cryogenic temperatures, above $3.2 \mathrm{eV}$, the $\mathrm{QE}$ slightly increased. This could be due to the increase in the energy loss in electron-electron scattering, and its reduced probability due to the larger band gap. The simulation shows that at $532 \mathrm{~nm}$, there is a QE drop of $50 \%$ at LN2 temperature, which is close to the analytical model. This smaller reduction compared to another experimental 
measurement in the $704 \mathrm{MHz}$ SRF gun may be due to the fact that we used room-temperature phonon energy instead of the cryogenic temperature.

The reduction in $\mathrm{QE}$ could be compensated for by the Schottky effect when the cathode is operated in a high-field environment. This compensation already was observed in preliminary measurements with a $\mathrm{K}_{2} \mathrm{CsSb}$ cathode in the $704 \mathrm{MHz}$ SRF gun, with an accelerating gradient of $11 \mathrm{MV} / \mathrm{m}$. More systematic measurements are planned.

\section{OTHER APPLICATIONS OF THE SIMULATION}

During the simulation process, the number of emitted electrons can be counted by time steps. By the end of emission, with the moving average function for each 50 particles, we obtained the temporal response shown in Fig. 12. For the typical $50 \mathrm{~nm}$ thickness cathode, the simulated response time is $170 \mathrm{fs}(90 \%$ of the electrons emitted) from the curve, which is in agreement with the sub-ps response time measured in Ref. [26]. When the cathode is thinner, the response time is shorter, as shown in Fig. 12, due to shorter diffusion time from the back of the cathode to emission. Thus, a thin $(\sim 1 \mathrm{~nm})$ multialkali cathode is a candidate electron source to generate an ultrashort bunch.

The effect of the thickness on the QE of the cathode was also investigated by the simulation as shown in Fig. 13. The laser's energy would not be fully absorbed if the thickness of the cathode is less than the absorption length of the cathode material. Based on our simulations for a thickness $\geq 20 \mathrm{~nm}$, the QE is independent of the cathode's thickness. If the cathode is thicker fractionally more photons may be absorbed, but these energetic electrons need to travel distances longer than the electrons' escape depth, resulting in diminishing returns. Furthermore, thicker cathodes usually have a rougher surface resulting in a larger emittance, especially in high accelerating fields [21].

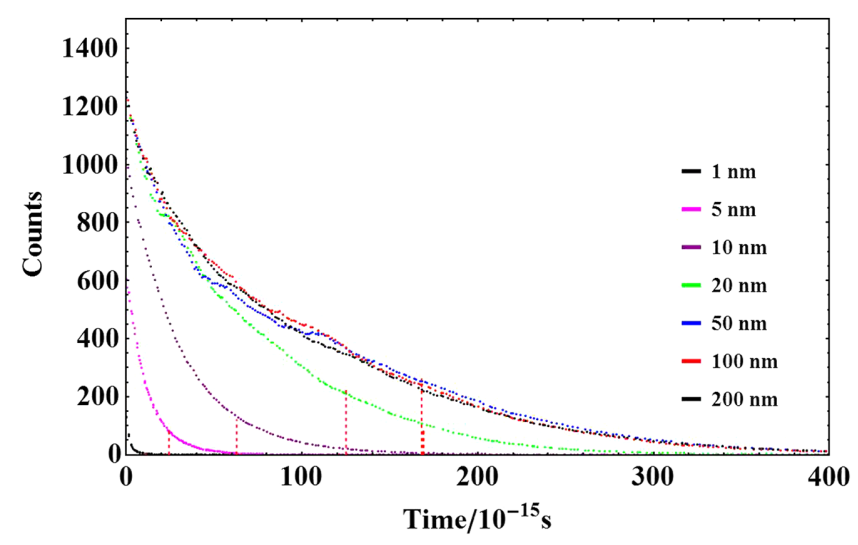

FIG. 12. Simulated temporal response of the $\mathrm{K}_{2} \mathrm{CsSb}$ cathode at room temperature with various thicknesses. The red dashed lines are the temporal response including $90 \%$ electrons.



FIG. 13. QE changes with different thicknesses. The dots are the experimental results of the $\mathrm{QE}$ of different thickness that matched the simulation very well.

However, a thicker cathode may prolong the lifetime [27]. Similar high QE ( 10\% at $532 \mathrm{~nm})$ has been obtained with a $20 \mathrm{~nm}$ thick cathode. The simulation result confirms that thickness has no effect on the QE when the cathode is thicker than $20 \mathrm{~nm}$.

\section{CONCLUSIONS}

Our experiments measure in detail the quantum efficiency as a function of photon energy and photocathode temperature, and show that the QE of bialkali photocathode inside the SRF gun will drop by at least 50\% if it is cooled down to the temperature of liquid nitrogen. We conclude that the increase in the ionization energy at low temperature is the main reason for this $\mathrm{QE}$ reduction. We built an analytical model that fits our data very well, and indicates increase in ionization energy and corresponding decrease in $\mathrm{QE}$ as the cathode temperature is lowered. A Monte Carlo simulation that includes electron scattering, and momentum conservation, was also developed. Both of the models fit our experimental data down to $166 \mathrm{~K}$. Extrapolation of the model beyond the range of the detailed experimental results indicate that the QE will decrease by $50 \%$ as the cathode's temperature is lowered from room temperature to that of liquid nitrogen, slightly underestimating the reduction in QE compared to the experimental results. During the initial beam experiment in BNL's $704 \mathrm{MHz}$ SRF gun, preliminary results indicate that the Schottky effect can partially compensate for this reduction in QE. Our Monte Carlo simulation provides additional information on the temporal response of the emission, the emittance as a function of photon energy, and the effect of cathode thickness on the quantum efficiency.

\section{ACKNOWLEDGMENTS}

This work was supported by Brookhaven Science Associates, LLC under Contract No. DE-SC0012704 with the U.S. Department of Energy. We would like to thank 
Dr. John Smedley for useful discussions on photoemission physics. We appreciate the useful discussions we had with Dr. Dimitre Dimitrov on developing the photoemission code. We appreciate the cooperation of the research group working on the ERL SRF gun who allowed us to be involved in testing our cathode in the $704 \mathrm{MHz}$ gun.

[1] F. Sannibale et al., Tests of photocathodes for high repetition rate $\mathrm{x}$-ray FELs at the APEX facility at LBNL, Proc. SPIE Int. Soc. Opt. Eng. 9512, 95121N (2015).

[2] L. Cultrera, S. Karkare, B. Lillard, A. Bartnik, I. Bazarov, B. Dunham, W. Schaff, and K. Smolenski, Growth and characterization of rugged sodium potassium antimonide photocathodes for high brilliance photoinjector, Appl. Phys. Lett. 103, 103504 (2013).

[3] J. Q. Xie et al., Real time evolution of antimony deposition for high performance alkali photocathode development, Proc. SPIE Int. Soc. Opt. Eng. 8847, 884705 (2013).

[4] D. H. Dowell et al., First operation of a photocathode radio frequency gun injector at high duty factor, Appl. Phys. Lett. 63, 2035 (1993).

[5] B. M. Dunham et al., Record high-average current from a high-brightness photoinjector, Appl. Phys. Lett. 102, 034105 (2013).

[6] W. Xu et al., First beam commissioning at BNL ERL SRF gun, in Proceedings of IPAC2015, Richmond, VA, 2015.

[7] T. Xin et al., Design of high-bunch-charge $112 \mathrm{MHz}$ superconducting rf photoemission electron source, Rev. Sci. Instrum. 87, 093303 (2016).

[8] S. Pastuszka, M. Hoppe, D. Kratzmann, D. Schwalm, A. Wolf, A. S. Jaroshevich, S. N. Kosolobov, D. A. Orlov, and A.S. Terekhov, Preparation and performance of transmission-mode GaAs photocathodes as sources for cold dc electron beams, J. Appl. Phys. 88, 6788 (2000).

[9] C. Ghosh and B. P. Varma, Preparation and study of properties of a few alkali antimonide photocathodes, J. Appl. Phys. 49, 4549 (1978).

[10] J. L. Shay, Temperature dependence of the energy gap in GaAs, Phys. Rev. B 4, 1385 (1971).

[11] Python 2.7 and 3.0 manual, http://www.python.org.

[12] E. Wang, T. Rao, and I. Ben-Zvi, Enhancement of photoemission from and postprocessing of $K_{2} \mathrm{CsSb}$ photocathode using excimer laser, Phys. Rev. ST Accel. Beams 17, 023402 (2014).

[13] L. Kalarasse, B. Bennecer, F. Kalarasse, and S. Djeroud, Pressure effect on the electronic and optical properties of the alkali antimonide semiconductors $\mathrm{Cs}_{3} \mathrm{Sb}, \mathrm{KCs}_{2} \mathrm{Sb}$,
$\mathrm{CsK}_{2} \mathrm{Sb}$ and $\mathrm{K}_{3} \mathrm{Sb}$ : Ab initio study, J. Phys. Chem. Solids 71, 1732 (2010).

[14] D. H. Dowell and J. F. Schmerge, Quantum efficiency and thermal emittance of metal photocathodes, Phys. Rev. ST Accel. Beams 12, 074201 (2009).

[15] W. E. Spicer, SLAC Report No. SLAC-PUB-6306, 1993.

[16] L. Cultrera, S. Karkare, H. Lee, X. Liu, I. Bazarov, and B. Dunham, Cold electron beams from cryocooled, alkali antimonide photocathodes, Phys. Rev. ST Accel. Beams 18, 113401 (2015).

[17] C. Jacoboni and L. Reggiani, The Monte Carlo method for the solution of charge transport in semiconductors with applications to covalent materials, Rev. Mod. Phys. 55, 645 (1983).

[18] A. R. H. F. Ettema and R. A. de Groot, Electronic structure of $\mathrm{Cs}_{2} \mathrm{KSb}$ and $\mathrm{K}_{2} \mathrm{CsSb}$, Phys. Rev. B 66, 115102 (2002).

[19] B. K. Chang, Y.Z. Liu, X. Dagang, Q. H. Zhan, and L. Zhou, Theoretical study on the thickness of polycrystalline semiconductor multialkali photocathodes, Proc. SPIE Int. Soc. Opt. Eng. 1982, 174 (1993).

[20] V. P. Beguchev, I. A. Shefova, and A. L. Musatov, Optical and photoemissive properties of multi-alkali photocathodes, J. Phys. D 26, 1499 (1993).

[21] S. Schubert et al., Bi-alkali antimonide photocathodes for high brightness accelerators, APL Mater. 1, 032119 (2013).

[22] K. L. Jensen, B. L. Jensen, E. J. Montgomery, D. W. Feldman, P. G. O'Shea, and N. Moody, Theory of photoemission from cesium antimonide using an alphasemiconductor model, J. Appl. Phys. 104, 044907 (2008).

[23] I. Bazarov, L. Cultrera, A. Bartnik, B. Dunham, S. Karkare, Y. Li, X. Liu, J. Maxson, and W. Roussel, Thermal emittance measurements of a cesium potassium antimonide photocathode, Appl. Phys. Lett. 98, 224101 (2011).

[24] Z. Zhao, A. Bartnik, F. W. Wise, I. V. Bazarov, and B. M. Dunham, High-power fiber lasers for photocathode electron injectors, Phys. Rev. ST Accel. Beams 17, 053501 (2014).

[25] M. B. Panish and H. C. Casey, Jr., Temperature dependence of the energy gap in GaAs and GaP, J. Appl. Phys. 40, 163 (1969).

[26] M. A. Nichols, Preparation/Characterization of Atomically Flat and Clean Mo (100) Surfaces and Thermal Emittance/ Response Time Measurements of $\mathrm{Cs}_{3} \mathrm{Sb}$ Photocathodes, http://www.lepp.cornell.edu/ ib38/reu/11/Nichols_report .pdf (2011).

[27] M. A. Mamun, C. Hernandez-Garcia, M. Poelker, and A. A. Elmustafa, Correlation of $\mathrm{CsK}_{2} \mathrm{Sb}$ photocathode lifetime with antimony thickness, APL Mater. 3, 066103 (2015). 